\title{
Triangular Lattice Model of Two-Dimensional Defect Melting
}

\author{
Jürgen Diete ${ }^{*}$ and Hagen Kleiner \\ Institut für Theoretische Physik, Freie Universität Berlin, Arnimallee 14, D-14195 Berlin, Germany
}

(Dated: Received November 21, 2018)

\begin{abstract}
We set up a harmonic lattice model for $2 \mathrm{D}$ defect melting which, in contrast to earlier simple-cubic models, lives on a triangular lattice. Integer-valued plastic defect gauge fields allow for the thermal generation of dislocations and disclinations. The model produces universal formulas for the melting temperature expressed in terms of the elastic constants, which are different from those derived for square lattices. They determine a Lindemann-like parameter for two-dimensional melting. In contrast to the square crystal which underwent a first-order melting transition, the triangular model melts in two steps. Our results are applied to the melting of Lennard-Jones and electron lattices.
\end{abstract}

PACS numbers: 61.72.Bb, 64.70.Dv, 64.90.+b

\section{INTRODUCTION}

Melting transitions 1, 2, 3] are of both technical and theoretical interest. Under suitable experimental circumstances one can study the melting process in two dimensions $(2 \mathrm{D})$. If the interparticle interactions are simple, the lowest-energy crystalline order in 2D is a close-packed triangular. It is observable for electrons on a liquid-helium surface [4], for adsorbed noble-gas atoms floating on an incommensurate substrate [5], for colloidal suspensions of highly charged spheres confined between parallel glass plates [] vortex lattices in thin films [8]. Simulation experiments provide us with further examples: rigid disks [9], Gaussian cores [10], and particles interacting with LennardJones 11] as well as Coulomb forces (Wigner lattice) 12].

There are various theories of $2 \mathrm{D}$ melting $2,3,13,14$, 15, 16, 17, 18]. Most popular is the phenomenological theory of Halperin, Nelson, and Young [14] which, inspired by the Kosterlitz and Thouless (KT) theory of vortices in superfluid films 19], explains the transition by the statistical behavior of defects. This KTHNY theory suggests that melting could proceed in a sequence of two continuous KT transitions, the first caused by the unbinding of dislocations, the second by the unbinding of disclinations. Such a sequence was indeed found in some $2 \mathrm{D}$ crystals experimentally and by simulations.

There are also explicit models on square lattices which allow to study defect melting in detail. These models are minimal in the sense that they contain the correct harmonic elastic energy and no anharmonic terms. The defects arise from integer-valued plastic gauge fields which account for the thermal creation and annihilation of dislocations and disclinations. The simplest such model shows only a single first-order melting transition 17]. Only after introducing an extra higher-gradient elastic term which gives the crystal a tunable angular stiffness, the first-order transition separates into two successive KT

\footnotetext{
*Electronic address: dietel@physik.fu-berlin.de
}

${ }^{\dagger}$ Electronic address: kleinert@physik.fu-berlin.de transitions [18]. This splitting can be observed in the laboratory, for instance in Xenon overlayer lattices on graphite [20].

Presently investigated systems live mostly on triangular lattices, and these seem to melt in two steps 6. 7, 8, 9, 21, 22, 23, 24], except for the Gaussian core model. The question arises whether a minimal melting model living on a triangular lattice will always undergo two successive transitions at the level of first gradient elastic energies only, so that no extra higher-gradient terms are needed for splitting them as on square lattices. It is the purpose of this paper to answer this question to the affirmative. We shall generalize the previously constructed lattice models from square lattices [2, 3, 17, 18] to triangular lattices and find, indeed, strong indications that they melt in two KT transitions.

A universal feature of all such defect models is that it is the combined separation of dislocations and disclinations which makes the melting transition first-order [17]. Dislocations alone would cause a similar phase transition as in superfluid helium, which undergoes a continuous transition.

An important virtue of the lattice defect models on square lattices was that they lead to a simple universal melting formula 2] determining the melting point in terms of the elastic constants. The result is obtained from a lowest-order approximation, which combines the high-temperature expansion of the defect contributions to the free energy density with the low-temperature expansion. The melting temperature is determined by the intersection of the two curves. This turns out to produce precisely the time-honored Lindemann criterion. In addition, however, there is a prediction of the universal value of the Lindemann number where melting occurs. The accuracy of the approximation was demonstrated quantitatively for square lattices in 3D and 2D 2, 25]. Recently, the results were successfully extended to face-centered and body-centered cubic lattices in 3D [26]. We shall find a similar formula for triangular lattices in 2D. The minimal lattice defect model will be constructed in Section II. From its partition function we calculate in Section III the melting temperature. In Section IV, we compare our result with the melting transition of Lennard-Jones and 
electron lattices. Section $\mathrm{V}$ discusses the melting values when taking into account nonlinear elasticity effects. In Section VI we use the model to predict a universal generalized Lindemann number 27] for 2D lattices.

\section{LATTICE HAMILTONIAN WITH DEFECTS}

The elastic energy of a crystal in the continuum approximation is given in $D$ dimensions by [28]

$$
E_{\mathrm{el}}=\int d^{D} x\left[\frac{\mu}{4}\left(\partial_{i} u_{j}+\partial_{j} u_{i}\right)^{2}+\frac{\lambda}{2}\left(\partial_{i} u_{i}\right)^{2}\right]
$$

where repeated indices are summed. The elastic constant $\mu$ is the shear modulus, and $\lambda$ the Lamé constant. In two dimensions, these constants are related to the elastic constants $c_{i j}$ with lattice symmetry by $\mu=c_{66}$ and $\lambda=$ $c_{11}-2 c_{66}$. The combination $c_{11}-c_{66}$ is the modulus of compression. The fields $u_{i}(x)(i=x, y)$ are the cartesian components of the atomic displacements.

In a first step we construct a Hamiltonian on a 2D triangular lattice whose continuum elastic energy agrees with (11). Of course, this construction is not unique. We restrict the freedom by the following extension requirements:

1. The lattice derivatives $\nabla_{x}$ and $\nabla_{y}$ must have the continuum limits $\partial_{x}$ and $\partial_{y}$, respectively.

2. $\nabla_{x}, \nabla_{y}$ should be maximally symmetric linear combinations of the nearest neighbor lattice derivatives $\nabla_{(l)}, l=1,2,3$ with respect to the point group of the lattice.

3. $\nabla_{x}$ and $\nabla_{y}$ should have the same transformation properties as $\partial_{x}$ and $\partial_{y}$ under the action of the point group of the lattice.

In the following we denote by $\mathbf{e}_{(l)}$ the oriented link vectors of the triangular lattice which surround a triangular face of the lattice. There are three such vectors:

$$
\begin{aligned}
& \mathbf{e}_{(1)}=\left(\cos \frac{2 \pi}{6}, \sin \frac{2 \pi}{6}\right), \quad \mathbf{e}_{(2)}=(-1,0), \\
& \mathbf{e}_{(3)}=\left(\cos \frac{2 \pi}{6},-\sin \frac{2 \pi}{6}\right) .
\end{aligned}
$$

and three conjugate vectors $\overline{\mathbf{e}}_{(l)}=-\mathbf{e}_{(l)}$

For each of the link vectors there exist a lattice derivative defined by

$$
\begin{aligned}
\nabla_{(1)} f(\mathbf{x}) & =\left[f\left(\mathbf{x}+\mathbf{e}_{(1)}\right)-f(\mathbf{x})\right] / a \\
\nabla_{(2)} f(\mathbf{x}) & =\left[f(\mathbf{x})-f\left(\mathbf{x}-\mathbf{e}_{(2)}\right)\right] / a \\
\nabla_{(3)} f(\mathbf{x}) & =\left[f\left(\mathbf{x}-\mathbf{e}_{(2)}\right)-f\left(\mathbf{x}+\mathbf{e}_{(1)}\right)\right] / a .
\end{aligned}
$$

where $a$ is the lattice spacing. In addition, there is a conjugate derivative $\bar{\nabla}_{(l)} f(\mathbf{x})$ defined by $-\nabla_{(l)} f(\mathbf{x})$ as in
Eq. (3), but with the replacement $\mathbf{e}_{(i)} \rightarrow \overline{\mathbf{e}}_{(i)}$. From the definition follows the identity

$$
\left(\nabla_{(1)}+\nabla_{(2)}+\nabla_{(3)}\right) f(\mathbf{x})=0
$$

for any function $f(\mathbf{x})$ on the lattice sites.

The 2D point group around each lattice face is given by $C_{3 v}$ [29]. This group causes permutations of the link directions $\mathbf{e}_{(l)}$ and the derivates $\nabla_{(l)}$. These can be represented by matrices which form a $3 \times 3$-dimensional representation of the point group $C_{3 v}$ in the space of linear combinations of the link vectors or of the lattice derivates.

We now define cartesian lattice versions $\nabla_{x}$ and $\nabla_{y}$ of the derivatives $\partial_{x}$ and $\partial_{y}$ as $C_{3 v^{-}}$symmetric linear combinations of the lattice derivatives $\nabla_{(l)}$ :

$$
\partial_{x, y} \rightarrow \nabla_{x, y} \equiv \frac{2}{3} e_{(l) x, y} \nabla_{(l)}
$$

Inserting the components from (2) these become

$$
\begin{aligned}
\partial_{x} \rightarrow \nabla_{x} & \equiv \frac{2}{3}\left[\left(\frac{1}{2} \nabla_{(1)}-\nabla_{(2)}+\frac{1}{2} \nabla_{(3)}\right)\right], \\
\partial_{y} \rightarrow \nabla_{y} & \equiv \frac{2}{3}\left[\left(\frac{\sqrt{3}}{2} \nabla_{(1)}-\frac{\sqrt{3}}{2} \nabla_{(3)}\right)\right] .
\end{aligned}
$$

It is easy to check that these fulfill the above requirements $1-3$.

Without proof we mention that by using group theoretical methods (see e.g. [29]) we can show that the extension requirements $1-3$ are sufficient to obtain unique lattice derivates $\nabla_{x}$ and $\nabla_{y}$. This is true for all other lattice symmetries. The only property needed for the proof is that the lattice possesses a link vector which generates all others by applying the symmetry group of the lattice.

\section{LATTICE HAMILTONIAN}

It is now easy to set up the lattice version of the continuum Hamiltonian (11). We simply replace the partial derivatives by the lattice derivatives (6), and obtain

$$
E_{\text {lat }}=\frac{v}{2} \sum_{\mathbf{x}}\left\{\frac{\mu}{2}\left[\nabla_{i} u_{j}(x)+\nabla_{j} u_{i}(x)\right]^{2}+\lambda\left[\nabla_{i} u_{i}(x)\right]^{2}\right\} .
$$

where the sum $\sum_{\mathbf{x}}$ runs over all lattice sites, and $v=$ $\sqrt{3} a^{2} / 2$ is the area of the fundamental cell of the lattice (for the square lattice $v=a^{2}$ ). As in the earlier work on square lattices [2] we shall first consider a symmetrized version of the Hamiltonian (7) which has the advantage of leading to explicit formulas for the melting transition for all $\mu, \lambda$. This arises by replacing $\nabla_{i}$ by $\bar{\nabla}_{i}$ in the $\lambda$ term:

$$
E_{\text {lat }} \approx \frac{v}{2} \sum_{\mathbf{x}}\left\{\frac{\mu}{2}\left[\nabla_{i} u_{j}(x)+\nabla_{j} u_{i}(x)\right]^{2}+\lambda\left[\bar{\nabla}_{i} u_{i}(x)\right]^{2}\right\} .
$$


Without an explicite discussion in this paper we mention that one can show the difference in the results using the symmetrized Hamiltonian (8) and the Hamiltonian (7) to be negligible for triangular as well as square lattices. The most symmetric way of writing (7) is

$$
\begin{aligned}
& E_{\text {lat }}=\frac{\sqrt{3} a^{2}}{4} \sum_{\mathbf{x}}\left\{\frac{4}{9} c_{11}\left(\sum_{j} \nabla_{(j)} u_{(j)}-\frac{1}{2} \sum_{i \neq j} \nabla_{(i)} u_{(j)}\right)^{2}\right. \\
& +\frac{1}{3} c_{66}\left(\sum_{k i j} \epsilon_{k i j} \nabla_{(i)} u_{(j)}\right)^{2} \\
& +\frac{2}{3} c_{66}\left(\sum_{k \neq i \neq j} 2 \nabla_{(k)} u_{(k)} \nabla_{(i)} u_{(j)}-2 \nabla_{(i)} u_{(k)} \nabla_{(k)} u_{(j)}\right. \\
& \left.\left.-\nabla_{(k)} u_{(k)} \nabla_{(i)} u_{(i)}+\nabla_{(i)} u_{(k)} \nabla_{(k)} u_{(i)}\right)\right\} .
\end{aligned}
$$

Here we have expanded the displacement vector in (7) in a symmetric way to $\mathbf{u}(x)=u_{(l)}(\mathbf{x}) \mathbf{e}_{(l)}$. This representation of $\mathbf{u}(\mathbf{x})$ is not unique due to the overcompleteness of the three link vectors (2). It becomes unique by setting $u_{(3)}=0$ and taking into account (4), yielding

$$
\begin{aligned}
& E_{\text {lat }}=\frac{\sqrt{3} a^{2}}{4} \sum_{\mathbf{x}}\left\{c_{11}\left(\nabla_{(1)} u_{(1)}+\nabla_{(2)} u_{(2)}\right)^{2}\right. \\
& +\frac{1}{3} c_{66}\left(\nabla_{(1)} u_{(1)}-\nabla_{(2)} u_{(2)}+2 \nabla_{(2)} u_{(1)}-2 \nabla_{(1)} u_{(2)}\right)^{2} \\
& \left.+4 c_{66}\left(\nabla_{(1)} u_{(2)} \nabla_{(2)} u_{(1)}-\nabla_{(1)} u_{(1)} \nabla_{(2)} u_{(2)}\right)\right\}
\end{aligned}
$$

\section{INCLUDING DEFECT GAUGE FIELDS}

With the goal of studying defect-induced melting transitions, the lattice representations (7) and (8) of linear elasticity must be extended by integer-valued defect gauge fields, the lattice version of plastic fields. From the textbook [2] we know how to do this for square lattices. The defect gauge fields reflect the fact that due to fluctuations, atoms are capable of exchanging positions with their neighbors and migrate eventually through the entire crystal. This process of self-diffusion makes it impossible to specify the displacement field uniquely. Thus, as a matter of principle, the displacement field is multivalued. It is determined only up to an arbitrary lattice vector, since it is impossible to say whether an atom is displaced by $\mathbf{u}(\mathbf{x})$ or by $\mathbf{u}(\mathbf{x})+a N_{(l)}(\mathbf{x}) \mathbf{e}_{(l)}$ where $N_{(l)}(\mathbf{x})$ is integer. The displacement vectors may have jumps across so-called Volterra surfaces which are described by integer-valued defect gauge fields $n_{(l m)}(\mathbf{x})$ in the lattice model [2]. These are, in general, not symmetric.

The defect gauge fields are included into the lattice derivatives of the Hamiltonian (7) by the replacement

$$
\nabla_{x, y} \mathbf{u}(\mathbf{x}) \rightarrow \frac{2}{3} e_{(l)}, y\left[\nabla_{(l)} \mathbf{u}(\mathbf{x})-n_{(l m)}(\mathbf{x}) \mathbf{e}_{(m)}\right]
$$

The classical partition functions of the triangular crystal including dislocation and disclination degrees of freedom is then given by

$$
Z=\prod_{\mathbf{x}, i}\left[\int_{-\infty}^{\infty} \frac{d u_{i}(\mathbf{x})}{a}\right] \sum_{\left\{n_{l m}(\mathbf{x})\right\}} \Phi\left[n_{(l m)}\right] \exp \left[-E_{\text {lat }} / k_{B} T\right] .
$$

For simplicity, we choose periodic boundary conditions for the displacement fields $\mathbf{u}(\mathbf{x})$. Since defects are spanned over an integer number of fundamental cells of the lattice, the functional $\Phi\left[n_{(l m)}\right]$ should restrict the range of the integer-valued gauge field $n_{(l m)}$. Furthermore, it acts also as a gauge-fixing, in such a way that only physically independent degrees of freedom are summed.

Due to the identity (44) we have the freedom to carry out the replacement $\nabla_{x, y} \rightarrow \nabla_{x, y}+a_{x, y}\left(\nabla_{(1)}+\nabla_{(2)}+\right.$ $\left.\nabla_{(3)}\right)$ in (6) with some constants $a_{x, y}$ without changing the elastic energy in (7) or (8). One can see that by considering relation (11), the resulting Hamiltonian and also the partition functions depends strongly on $a_{x, y}$. This is because the replacement (11) does not take into account the fact that defect lines as dislocations and disclinations are built of interstitials or vacancies, respectively, which allows only one interstitial or vacancy per fundamental cell. This restricts the Volterra surfaces to run through an integer number of fundamental cells. (See Fig. 1). This restriction on the defect gauge fields results in the following constraint

$$
n_{(1 l)}(\mathbf{x})+n_{(2 l)}(\mathbf{x})+n_{(3 l)}(\mathbf{x})=0,
$$

for all $l=1,2,3$. Here we take into account that for a dislocation the jump over the Volterra surface is constant. Disclinations are built of dislocations within our lattice model. By taking into account this constraint on the defect gauge fields the resulting partition function does not longer depend on the replacement constants $a_{x, y}$.

We now must identify and eliminate the gauge degrees of freedom in Eq. (12). The overcompleteness of the three basis vectors $\mathbf{e}_{(l)}$ of the two-dimensional lattice implies that the replacement (11) leads to the same defects if the integer numbers satisfy $n_{(l i)}(\mathbf{x}) \mathbf{e}_{(i)}=0$ for $l=1,2,3$. The rest of the gauge freedoms can be eliminated by analogy with the gauge fixing on the square lattice in the textbook [2]. Initially, the displacement fields cover only a single fundamental cell. We can extend the range to the entire crystal by fixing two of the last four components of the defect gauge field $n_{(l m)}$. One more component can be eliminated since $E_{\text {lat }}$ depends on the derivates $\nabla_{x} u_{y}$ and $\nabla_{y} u_{x}$ via the sum $\nabla_{y} u_{x}+\nabla_{x} u_{y}$.

Finally, we must take care of the constraints due to the periodic boundary conditions. The technical aspects of the elimination of the gauge degrees of freedom are deferred to Appendix A. Eventually we arrive at the par- 


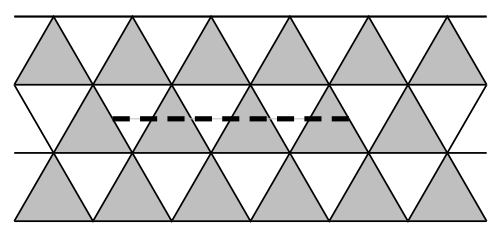

FIG. 1: Cut in a triangular lattice. For each lattice site, the shaded regions show the faces which are surrounded by the oriented link vectors (2) associated with the lattice derivates $\nabla_{(l)}$ in Eq. (3). Dashed line indicates a Volterra surface crossing an integer number of fundamental cells.

tition function:

$$
\begin{aligned}
& Z=\prod_{\mathbf{x}, i}\left[\int_{-\infty}^{\infty} \frac{u_{i}(\mathbf{x})}{a} \sum_{n(\mathbf{x})=-\infty}^{\infty}\right] \\
& \times\left(\begin{array}{c}
\left(1-\delta_{\mathbf{x}, \mathcal{B}}\right)+\delta_{\mathbf{x}, \mathcal{B}} \\
n_{(11)}(\mathbf{x}), n_{(21)}(\mathbf{x})=-\infty
\end{array}\right) \exp \left[-E_{\text {lat }} / k_{B} T\right]
\end{aligned}
$$

with the lattice energy (7) and the replacements of the lattice derivatives

$$
\begin{aligned}
&\left(\nabla_{x}, \nabla_{y}\right) \times \mathbf{u}(\mathbf{x}) \rightarrow \frac{2}{3}\left(e_{(l)_{x}}, e_{(l)}\right) \times \nabla_{(l)} \mathbf{u}(\mathbf{x}) \\
&+\left(\begin{array}{cc}
0 & \frac{1}{\sqrt{3}} n(\mathbf{x}) \\
\frac{1}{\sqrt{3}} n(\mathbf{x}) & 0
\end{array}\right) \\
& \quad+\delta_{\mathbf{x}, \mathcal{B}} \frac{1}{2}\left(\begin{array}{cc}
n_{(21)}(\mathbf{x}) & -\frac{1}{\sqrt{3}} n_{(21)}(\mathbf{x})-\frac{2}{\sqrt{3}} n_{(11)} \\
+\sqrt{3} n_{(21)}(\mathbf{x}) & -2 n_{(11)}(\mathbf{x})-n_{(21)}(\mathbf{x})
\end{array}\right)
\end{aligned}
$$

where $\mathcal{B}$ contains the sites at the rightmost and top boundary of the sample.

The gauge degrees of freedoms are fixed by choosing $n(\mathbf{x})=0$ for $\mathbf{x} \in \mathcal{B}$, further $\nabla_{x} n(\mathbf{x})=0$ on the rightmost boundary $\mathcal{B}_{r}$, and $\nabla_{y} n(\mathbf{x})=0$ on the topmost boundary $\mathcal{B}_{t}$ of the model. This will be summarized by the statement

$$
n(\mathbf{x})=0 \quad \text { for } \quad \mathbf{x} \in \mathcal{B}^{\prime}
$$

where $\mathcal{B}^{\prime}$ is the extended boundary consisting on the sites $\mathcal{B}, \mathcal{B}_{t}+a \mathbf{e}_{(1)}$ and $\mathcal{B}_{r}+a \mathbf{e}_{(2)}$. The boundary fields $n_{(11)}(\mathbf{x})$ and $n_{(21)}(\mathbf{x})$ in (15) result from the periodic boundary conditions of the displacement field $\mathbf{u}$, which restrict the gauge degrees of freedom.

The final partition function (14) describes a triangular crystal with harmonic elastic fluctuations and fluctuating dislocations and disclinations.

By a standard duality transformation it is possible to rewrite this partition function in a canonical form. Introducing the conjugate stress fields $\sigma_{i j}(\mathbf{x})$ via an auxiliary integral, we see that (14) is equal to

$$
\begin{aligned}
& Z=\left[\frac{\mu}{4(\lambda+\mu)}\right]^{N / 2}\left(\frac{1}{2 \pi \beta_{\triangle}}\right)^{3 N / 2} \prod_{\mathbf{x}, i \leq j}\left[\int_{-\infty}^{\infty} d \sigma_{i j}\right] \\
& \times \prod_{\mathbf{x}}\left[\sum_{n(\mathbf{x})=-\infty}^{\infty} \int_{-\infty}^{\infty} \frac{d \mathbf{u}(\mathbf{x})}{a}\right] \exp \left\{-\frac{1}{2 \beta} \sum_{\mathbf{x}}\right. \\
& \left.\times\left[\sum_{i<j} \sigma_{i j}^{2}+\frac{1}{2} \sum_{i} \sigma_{i i}^{2}-\frac{\lambda}{4(\lambda+\mu)}\left(\sum_{i} \sigma_{i i}\right)^{2}\right]\right\} \\
& \times \exp \left[i 2 \pi \sum _ { \mathbf { x } } \frac { 2 } { 3 } \left[\sum_{i} e_{(l) i} \nabla_{(l)} u_{i} \sigma_{i i}\right.\right. \\
& \left.\left.+\left(e_{(l)} \nabla_{(l)} u_{2}+e_{(l)} \nabla_{(l)} u_{1}\right) \sigma_{12}\right]+H_{\sigma n}\right]
\end{aligned}
$$

where $\beta_{\triangle} \equiv a^{2} \mu \sqrt{3} / 2 k_{B} T(2 \pi)^{2}$. For details see [2].

The energy $H_{\sigma n}$ couples the stress field $\sigma_{i j}$ to the integer value fields $n_{(i j)}(\mathbf{x})$ :

$$
\begin{aligned}
& H_{\sigma n}=i 2 \pi \sum_{\mathbf{x}} \frac{2}{\sqrt{3}} \sigma_{12} n(\mathbf{x})+\ln \left[\prod_{\mathbf{x} \in \mathcal{B}}\left[\sum_{n_{(11)}, n_{(21)}(\mathbf{x})=-\infty}^{\infty}\right]\right. \\
& \exp \left[i 2 \pi \left(\frac{1}{2} \sigma_{(11)}(\mathbf{x}) n_{(21)}(\mathbf{x})-\frac{1}{2} \sigma_{(22)}(\mathbf{x})\left(n_{(21)}(\mathbf{x})+\right.\right.\right. \\
& \left.\left.\left.\left.+2 n_{(11)}(\mathbf{x})\right)+\frac{1}{\sqrt{3}} \sigma_{(12)}(\mathbf{x})\left(n_{(21)}(\mathbf{x})-n_{(11)}(\mathbf{x})\right)\right)\right]\right]
\end{aligned}
$$

This is the dual representation of (14). For very large lattices, the integer-valued fields $n_{(11)}(\mathbf{x}), n_{(21)}(\mathbf{x})$ have, of course, no physical implications since they exists only on the boundary of the system. For the present calculations on a finite lattice they are, however, needed.

The partition function (17) corresponds to the Hamiltonian (7) including defects. The partition function for the symmetrized Hamiltonian (8) is given by the same expression with the replacement

$$
\frac{\lambda}{4(\lambda+\mu)}\left(\sum_{i} \sigma_{i i}\right)^{2} \rightarrow \frac{\lambda}{4(\lambda+\mu)}\left(\sum_{i} \frac{\bar{\nabla}_{i}}{\nabla_{i}} \sigma_{i i}\right)^{2}
$$

in the third term of the first exponent in (17). In the following sections, we shall use the symmetrized Hamiltonian (8) corresponding to the partition function (17) with (19) as the basis for calculating physical quantities.

\section{MELTING TRANSITION}

We now determine the melting temperature of the model. 


\section{A. Lowest-Order Approximation}

It has been shown in the textbook [2] that the intersection of the free energies of the two limiting curves of the high- and low- temperature expansion yields good estimates for the melting temperatures for simple cubic lattices in three dimensions and for square lattices in two dimensions (see Figs. 12.1 and 12.2 on pp. 1084-1085).

For small $T$, the defects are frozen out, and the partition function has the classical limit of the partition function of the Hamiltonian (8) with the derivate substitution (11) and $n(\mathbf{x}) \equiv 0$ :

$$
Z_{T \rightarrow 0}=\left(2 \pi \beta_{\triangle}\right)^{-N}\left(\frac{\mu}{\lambda+2 \mu}\right)^{N / 2} e^{-N \ell_{\triangle}} .
$$

The parameter $\ell_{\triangle}$ denotes the trace of the logarithm of the triangular lattice Laplacian divided by the number of sites. The Fourier transforms of the lattice derivates (3) are

$$
\begin{aligned}
& K_{(1)}=\frac{1}{i a}\left(e^{i a \mathbf{k} \cdot \mathbf{e}_{(1)}}-1\right), \quad K_{(2)}=\frac{1}{i a}\left(1-e^{-i a \mathbf{k} \cdot \mathbf{e}_{(2)}}\right) \\
& K_{(3)}=\frac{1}{i a}\left(e^{-i a \mathbf{k} \cdot \mathbf{e}_{(2)}}-e^{i a \mathbf{k} \cdot \mathbf{e}_{(1)}}\right) .
\end{aligned}
$$

The conjugate lattice derivatives have the complexconjugate Fourier transforms $\bar{K}_{(l)}=K_{(l)}^{*}$. Using these, we calculate $\ell_{\triangle}$ from the momentum integral

$$
\begin{aligned}
\ell_{\triangle} & =\frac{1}{2 A_{\mathrm{BZ}}} \int_{\mathrm{BZ}} d^{2} k \log \left(\left[\frac{4 a^{2}}{9} \bar{K}_{(l)} K_{(m)} \mathbf{e}_{(l)} \cdot \mathbf{e}_{(m)}\right]^{2}\right) \\
& \approx 1.22 .
\end{aligned}
$$

More explicitly, the argument of the logarithm is

$$
\begin{aligned}
& \frac{4 a^{2}}{9} \bar{K}_{(l)} K_{(m)} \mathbf{e}_{(l)} \cdot \mathbf{e}_{(m)} \\
& =\left[4-\frac{4}{3}\left(\cos a \mathbf{k} \cdot \mathbf{e}_{(1)}+\cos a \mathbf{k} \cdot \mathbf{e}_{(2)}+\cos a \mathbf{k} \cdot \mathbf{e}_{(3)}\right)\right]
\end{aligned}
$$

The momentum integral in (23) runs over the $2 \mathrm{D}$ Brioullin zone whose area is $A_{\mathrm{BZ}}=(2 \pi)^{2} \sqrt{3} / 2 a^{2}$. Let us compare the value (22) with the corresponding on the square lattice in Ref. 2], where the low-temperature partition function looks like (20), but with $\ell_{\triangle} \approx 1.22$ replaced by $\ell_{\square} \approx 1.14$, and $\beta_{\triangle} \equiv a^{2} \mu \sqrt{3} / 2 k_{B} T(2 \pi)^{2}$ by $\beta_{\square} \equiv a^{2} \mu / k_{B} T(2 \pi)^{2}$.

Above the melting point, the partition function is calculated from the dual representation (17). In the hightemperature limit, the defects are prolific. The sum over $n(\mathbf{x})$ enforces $\sigma_{12}(\mathbf{x})$ to have discrete values such that terms with $\sigma_{12}(\mathbf{x}) \neq 0$ are exponentially small for large temperature. Thus we can restrict ourselves to terms with $\sigma_{12}(\mathbf{x})=0$ only. After carrying out the integrals over the lattice displacements $\mathbf{u}(\mathbf{x})$, we obtain the constraint that the stress field is divergenceless. Its indepen- dent components are integrated out using the formulas

$$
\begin{aligned}
& \prod_{\mathbf{x}}\left[\int_{-\infty}^{\infty} d \sigma_{11}\right] \delta\left(\frac{2 a}{3} e_{(l)_{i}} \bar{\nabla}_{(l)} \sigma_{i 1}(\mathbf{x})\right) \\
& =\exp \left[-\frac{N}{2 A_{\mathrm{BZ}}} \int_{\mathrm{BZ}} d^{2} k \log \left(\frac{4 a^{2}}{9} \bar{K}_{(l)} K_{(m)} e_{(l)_{x}} e_{(m)_{x}}\right)\right] \\
& =\exp \left[-\frac{N}{2 A_{\mathrm{BZ}}} \int_{\mathrm{BZ}} d^{2} k \log \left(2-2 \cos a \mathbf{k} \cdot \mathbf{e}_{(2)}\right)\right]=1
\end{aligned}
$$

and

$$
\begin{aligned}
& \prod_{\mathbf{x}}\left[\int_{-\infty}^{\infty} d \sigma_{22}\right] \delta\left(\frac{2 a}{3} e_{(l)} \bar{\nabla}_{(l)} \sigma_{i 2}(\mathbf{x})\right) \\
& =\exp \left[-\frac{N}{2 A_{\mathrm{BZ}}} \int_{\mathrm{BZ}} d^{2} k \log \left(\frac{4 a^{2}}{9} \bar{K}_{(l} K_{(m)} e_{(l)} e_{(m)}\right)\right] \\
& =\exp \left\{-\frac{N}{2 A_{\mathrm{BZ}}} \int_{\mathrm{BZ}} d^{2} k \log \left[\frac { 1 } { 3 } \left(6-4 \cos a \mathbf{k} \cdot \mathbf{e}_{1}\right.\right.\right. \\
& \left.\left.\left.\quad-4 \cos a \mathbf{k} \cdot \mathbf{e}_{3}+2 \cos a \mathbf{k} \cdot \mathbf{e}_{2}\right)\right]\right\} \approx\left(\frac{1}{1.15}\right)^{N} .
\end{aligned}
$$

The partition function $Z$ in this limit is

$$
Z_{T \rightarrow \infty}=\left(2 \pi \beta_{\triangle}\right)^{-3 N / 2}\left[\frac{\mu}{4(\lambda+\mu)}\right]^{N / 2} C_{\triangle}^{N / 2},
$$

where $C_{\triangle}$ is the constant

$$
C_{\triangle} \approx 0.57
$$

From the intersection of the high-temperature expansion (26) with the the low-temperature expansion (20) we obtain the lowest-order result for the melting transition of a triangular lattice

$$
\beta_{\triangle}(1+\nu) \approx \frac{1}{4 \pi} e^{2 \ell_{\triangle}} C_{\triangle} \approx 0.52
$$

where $(1+\nu)=2(\lambda+\mu) /(\lambda+2 \mu)$. This is somewhat smaller than $\beta_{\square}(1+\nu) \approx 0.81$ for the square lattice [2].

Let us now see how these results are changed by a systematic improvement of the lowest-order low- and hightemperature expansions (20) and (26).

\section{B. Beyond the Lowest Order}

In the low-temperature regime, we include the leading contributions with nonzero defect gauge fields $n(\mathbf{x})$ in the partition function (14). In the dual representation (17), we must take into account the integer nature of $n(\mathbf{x})$. This was done in Ref. [2] by reexpressing (17) in terms of the physical defect density field, which is the double-curl of the defect gauge field. Here we carry out a similar calculation for the triangular lattice. After performing, in (17), the integrals over the displacement field $\mathbf{u}(\mathbf{x})$ and the stress fields $\sigma_{i j}(\mathbf{x})$ we obtain the improved 
low-temperature approximation $Z=Z_{T \rightarrow 0} Z_{\triangle}^{\text {def }}$ with the defect correction factor

$$
\begin{aligned}
& Z_{\triangle}^{\text {def }}=\prod_{\mathbf{x}} \sum_{n(\mathbf{x})=-\infty}^{\infty}\left(\left(1-\delta_{\mathbf{x}, \mathcal{B}}\right)+\delta_{\mathbf{x}, \mathcal{B}} \sum_{n_{(12)}, n_{(11)}(\mathbf{x})=-\infty}^{\infty}\right) \\
& \times \exp \left[-4 \pi^{2} \beta_{\triangle}(1+\nu) \sum_{\mathbf{x}, \mathbf{x}^{\prime}} \frac{4 a^{2}}{9} \nabla_{(l)} e_{(l) x} \nabla_{(m)} e_{(m) y} \tilde{n}(\mathbf{x})\right. \\
& \left.\times v_{\triangle}\left(\mathbf{x}-\mathbf{x}^{\prime}\right) \frac{4 a^{2}}{9} \nabla_{(k)} e_{(k) x} \nabla_{(n)} e_{(n) y} \tilde{n}\left(\mathbf{x}^{\prime}\right)\right]
\end{aligned}
$$

and

$$
\begin{aligned}
& \tilde{n}(\mathbf{x})=\frac{2}{\sqrt{3}} n(\mathbf{x})+\left(\frac{\nabla_{(l)} e_{(l)_{x}}}{\nabla_{(l)} e_{(l)_{y}}}-\frac{1}{\sqrt{3}}\right) n_{(11)}(\mathbf{x}) \delta_{\mathbf{x}, \mathcal{B}} \\
& +\left(\frac{1}{\sqrt{3}}-\frac{1}{2} \frac{\nabla_{(l)} e_{(l)_{y}}}{\nabla_{(l)} e_{(l)_{x}}}+\frac{1}{2} \frac{\nabla_{(l)} e_{(l)_{x}}}{\nabla_{(l)} e_{(l)_{y}}}\right) n_{(21)}(\mathbf{x}) \delta_{\mathbf{x}, \mathcal{B}} .
\end{aligned}
$$

The numbers $n(\mathbf{x})$ fulfill the boundary condition (16). In this way, we have expressed the low-temperature corrections to the harmonic partition function as a partition function of integer-valued fields $n(\mathbf{x})$ in the bulk and two integer valued fields $n_{(11)}(\mathbf{x}), n_{(21)}(\mathbf{x})$ on the boundary of the sample.

The interaction potential $v_{\triangle}$ is given by the inverse square of the triangular Laplacian

$$
v_{\triangle}(\mathbf{x})=\left[\frac{4 a^{2}}{9} \nabla_{(l)} \nabla_{(m)} \mathbf{e}_{(l)} \cdot \mathbf{e}_{(m)}\right]^{-2}(\mathbf{x}) \equiv \frac{1}{a^{4}} \square_{\triangle}^{-2}(\mathbf{x}) .
$$

It is not easy to obtain the leading-order contributions of $Z_{\triangle}^{\text {def }}$. This is mainly due to cancellation effects in the configuration sum coming from the interaction of the fields $n(\mathbf{x})$ and $n_{(11)}(\mathbf{x}), n_{(21)}(\mathbf{x})$ on the boundary of the sample. A more efficient calculation is possible if we go over from the defect gauge fields to the defect density field given by

$$
\eta(\mathbf{x})=\frac{2 a^{2}}{3} \epsilon_{k l} \epsilon_{m n} \nabla_{k} \nabla_{m} e_{(i)} n_{(i j)}(\mathbf{x}) e_{(j)_{n}} .
$$

The defect field $\eta(\mathbf{x})$ describes disclination degrees of freedoms [2]. The dislocations arise from dipoles of two nearby disclinations. The main advantage of $\eta(\mathbf{x})$ is that it is invariant under defect gauge transformations of the integer-valued fields $n_{(i j)}$. Thus, we obtain for $Z_{\triangle}^{\text {def }}$ of Eq. (29):

$$
Z_{\triangle}^{\text {def }}=\prod_{\mathbf{x}} \sum_{\eta(\mathbf{x})} e^{-4 \pi^{2} \beta_{\triangle}(1+\nu) \eta(\mathbf{x}) v_{\triangle}\left(\mathbf{x}-\mathbf{x}^{\prime}\right) \eta(\mathbf{x})} .
$$

The sum $\sum_{\eta(\mathbf{x})}$ runs over all different field configurations $\eta(\mathbf{x})$ defined by (33). The values for $v_{\triangle}(\mathbf{x})$ can be calculated via the Fourier transform

$$
v_{\triangle}(\mathbf{x})=\frac{1}{A_{\mathrm{BZ}}} \int_{\mathrm{BZ}} d^{2} k \frac{\exp (i \mathbf{k x})}{\left[\frac{4 a^{2}}{9} \bar{K}_{(l)} K_{(m)} \mathbf{e}_{(l)} \cdot \mathbf{e}_{(m)}\right]^{2}} .
$$

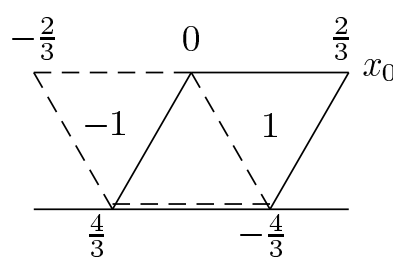

(a)

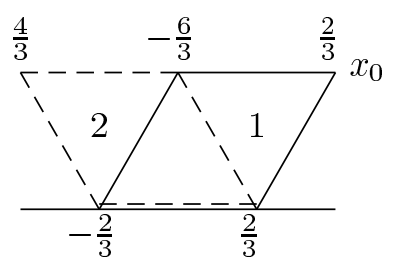

(b)
FIG. 2: Basic configurations of defect density $\eta(\mathbf{x})$ associated with the defect gauge field $n_{(i j)}(\mathbf{x})=\delta_{i, 1} \delta_{j, 2} \delta_{\mathbf{x}, \mathbf{x}_{0}}$ (a), and $n_{(i j)}(\mathbf{x})=\delta_{i, 1} \delta_{j, 1} \delta_{\mathbf{x}, \mathbf{x}_{0}}$ (b). The numbers in the triangles denote the multiplicity of basic charges $2 / 3$.

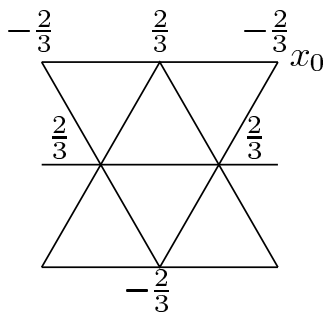

FIG. 3: Defect configuration $\eta(\mathbf{x})$ for $n_{(i j)}(\mathbf{x})=\left(\delta_{i, 2} \delta_{j, 1}-\right.$ $\left.\delta_{i, 1} \delta_{j, 2}-\delta_{i, 1} \delta_{j, 1}\right) \delta_{\mathbf{x}, \mathbf{x}_{0}}$. Its contribution to the free energy is given by the first term in Eq. (36).

We fix the gauge freedom due to overcounting by setting $n_{l 3}=0$. Upon further using (13) we can express $\eta(\mathbf{x})$ in terms of the fields $n_{(i j)}$ with $i, j=1,2$ only. The explicite relations are given in Eqs. (A1) and (A2). Figure 2(a) illustrates the non-zero $\eta(\mathbf{x})$ values for $n_{(i j)}(\mathbf{x})=\delta_{i, 1} \delta_{j, 2} \delta_{\mathbf{x}, \mathbf{x}_{0}}$, while $2(\mathrm{~b})$ does the same thing for $n_{(i j)}(\mathbf{x})=\delta_{i, 1} \delta_{j, 1} \delta_{\mathbf{x}, \mathbf{x}_{0}}$. The cases $n_{(i j)}(\mathbf{x})=$ $\delta_{i, 2} \delta_{j, 1} \delta_{\mathbf{x}, \mathbf{x}_{0}}$ and $n_{(i j)}(\mathbf{x})=\delta_{i, 2} \delta_{j, 2} \delta_{\mathbf{x}, \mathbf{x}_{0}}$ follow from linear combinations of $2(\mathrm{a})$ and $2(\mathrm{~b})$ after a clockwise rotation by an angle $2 \pi / 3$. The space of all $\eta$-fields are spanned by linear combinations of these four configurations modulo translations. The figure shows that the values of the $\eta$-field are multiples of $2 / 3$ which we call defect charges. All $\eta$-configurations are composed of two neutral rhombuses which are illustrated by the dashed and straight lines in Fig 2. Besides being neutral, the rhombuses have also no dipole moments. The figure shows the multiplicity of the basic charges for both rhombuses. The fact that the $\eta$-fields are neutral and dipole-free agrees with the situation on the square lattice. There is one difference, however. On the triangular lattice there are neutral dipole-free charge configurations composed of multiples of basic charges $2 / 3$ which cannot be obtained from an $\eta$-field. One simple example is a single basic rhombus shown by the dashed line in Fig. 2(a) built of defect charges $\pm 2 / 3$. The complication with respect to the square lattice where the $\eta$-fields are built of all neutral and dipole-free charge configurations is due to the constraint (13). Finally we mention that the basic configuration $\tilde{n}(\mathbf{x})=(2 / \sqrt{3}) \delta_{\mathbf{x}, \mathbf{x}_{0}}$ in (30) 
with $\eta(\mathbf{x})=-a^{2} \nabla_{x} \nabla_{y} \tilde{n}(\mathbf{x})$ for $\mathbf{x} \notin \mathcal{B}$ corresponds to the defect configuration $2(\mathrm{a})$.

We shall now prove that all localized $\eta(\mathbf{x})$ fields can be built from localized $n_{(i j)}(\mathbf{x})$-fields if the sample is infinite. This can be seen from the fact that the line $\mathcal{B}$ in (29) and (30) can be moved also to the middle of the system where we held fixed the boundary $\mathcal{B}^{\prime}$ in (16). This follows immediately from the considerations in Appendix A and the periodic boundary condition of the displacement fields $u_{i}(\mathbf{x})$ used here. Thus, we have to take into account only a linear combination of the most localized fields $n_{(i j)}(\mathbf{x})$ in (33) to get the leading order in the defect partition function (34).

Writing $Z_{\triangle}$ as an exponential of the connected diagrams $Z=e^{-\beta F}$, we find the free energy of the defect fields

$-F_{\triangle}^{\mathrm{def}} / k_{B} T \approx 2 N e^{-4 \pi^{2} \tilde{v} \triangle(0) \beta_{\triangle}(1+\nu)}+6 N e^{-4 \pi^{2} \tilde{v}_{\triangle}(0) \beta_{\triangle} \frac{4(1+\nu)}{3}}$

where $\nabla_{x}, \nabla_{y}$ are the triangular lattice derivates in (6) and

$$
\tilde{v}_{\triangle}(\mathbf{x}-\mathbf{x})=a^{4} \nabla_{x} \nabla_{y} \nabla_{x^{\prime}} \nabla_{y^{\prime}} v_{\triangle}\left(\mathbf{x}-\mathbf{x}^{\prime}\right) .
$$

By carrying out a numerical integration of the Fourier transform similar to (22) we obtain

$$
\tilde{v}_{\triangle}(0) \approx 0.163, \quad \tilde{v}_{\triangle}\left(a \mathbf{e}_{(i)}\right) \approx-0.03
$$

These numbers are found by numerical integration of the Fourier representation similar to (35). The first term in (36) corresponds to the $\eta(\mathbf{x})$-configuration in Fig. 3 . The second term in (36) corresponds to the $\eta$-configuration 2 (a) in Fig. 2. The factor $6 N$ comes from the six possibilities to cover this basic $\eta$-configuration on the lattice, this means the configuration $2(\mathrm{a})$, the rotated configuration by angle $2 \pi / 3$ and $4 \pi / 3$ plus the negative of all these three configurations. The other basic diagrams are approximately a factor $\exp \left[-4 \pi^{2} v_{\triangle}(0) \beta_{\triangle}(1+\nu)\right]$ smaller than the leading terms in (36). When using the meanfield value $\beta_{\triangle}(1+\nu) \approx 0.6$ we obtain additive corrections which approximatively a factor 0.04 smaller than the leading terms in $-F_{\triangle}^{\text {def }} / k_{B} T$ calculated above. From this, we derive also a temperature regime for which our lowest defect configuration result can be trusted. This is given by the inequality $4 \pi^{2} v_{\triangle}(0) \beta_{\triangle}(1+\nu)>1$ resulting in $\beta_{\triangle}(1+\nu)>0.15$.

Recall that for square lattices, the defect correction factor looks similar to (36), except that the second term is missing, and that $\tilde{v}_{\triangle}$ in the first term is replaced by $\tilde{v}^{\square}$, where $\tilde{v}^{\square}$ is defined by Eq. (37), with $v^{\square}$ being the inverse square of the Euclidean lattice Laplacian 2 . The numerical value is $\tilde{v}^{\square}(0) \approx 0.16[2]$.

Next, we consider the higher-order correction factor $Z^{\text {stress }}$ to the high-temperature expansion of the partition function $Z=Z_{T \rightarrow \infty} Z^{\text {stress }}$. The correction factor is obtained by carrying out the integration over the displacement field $\mathbf{u}(\mathbf{x})$ in Eq. (17), which makes $\sigma_{12}(\mathbf{x})$ discretevalued, and further summing over the defect gauge fields $n_{(i j)}(\mathbf{x})$. The result is

$$
\begin{aligned}
& Z_{\triangle}^{\text {stress }}=\prod_{\mathbf{x}}\left(\left(1-\delta_{\mathbf{x}, \mathcal{B}^{\prime}}\right)\left[\sum_{\sigma_{12}(\mathbf{x}) \in \sqrt{3} \mathbb{Z} / 2}\right]\right.
\end{aligned}
$$

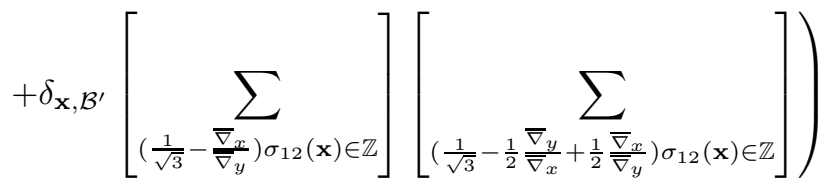

$$
\begin{aligned}
& \times \exp \left[\frac { - 1 } { 4 \beta _ { \triangle } ( 1 + \nu ) } \sum _ { \mathbf { x } , \mathbf { x } ^ { \prime } } \sigma _ { 1 2 } ( \mathbf { x } ) \left[\frac{4 a^{2}}{9} \bar{\nabla}_{(l)} \mathbf{e}_{(l) x} \bar{\nabla}_{(m)} \mathbf{e}_{(m) y}\right.\right. \\
& \left.\left.\times v_{\triangle}\left(\mathbf{x}-\mathbf{x}^{\prime}\right) \frac{4 a^{2}}{9} \nabla_{(k)} \mathbf{e}_{(k) x^{\prime}} \nabla_{(n)} \mathbf{e}_{(n) y^{\prime}}\right]^{-2} \sigma_{12}\left(\mathbf{x}^{\prime}\right)\right] \text {. }
\end{aligned}
$$

Most of the $\sigma_{12}$ configurations give a zero contribution to $Z_{\triangle}^{\text {stress }}$ in the sum $\sum_{\sigma_{12}(\mathbf{x}) \in \sqrt{3} \mathbb{Z} / 2}$ in (39). We can extract the non-zero contributions by going to the gauge field $\chi(\mathbf{x})$ with the definition $\sigma_{12}(\mathbf{x})=a^{2} \nabla_{x} \nabla_{y} \chi(\mathbf{x})$. In order to get the summation values of the fields $\chi(\mathbf{x})$ we have to determine the lowest value $z$ such that $z a^{2} \nabla_{x} \nabla_{y} \delta_{\mathbf{x}, \mathbf{x}_{0}} \in$ $\sqrt{3} \mathbb{Z} / 2$ for an arbitrary lattice position $\mathbf{x}_{0}$. This value is given by $z=3 / 2$. Neglecting boundary terms, we obtain

$$
\begin{aligned}
& Z_{\triangle}^{\text {stress }}=\prod_{\mathbf{x}}\left[\sum_{\chi(\mathbf{x}) \in 3 \mathbb{Z} / 2}\right] \\
& \times \exp \left[-\frac{1}{4 \beta_{\triangle}} \frac{1}{(1+\nu)} \sum_{\mathbf{x}, \mathbf{x}^{\prime}} \chi(\mathbf{x})\left(v_{\triangle}\right)^{-2}\left(\mathbf{x}-\mathbf{x}^{\prime}\right) \chi\left(\mathbf{x}^{\prime}\right)\right]
\end{aligned}
$$

where $v_{\triangle}^{-2}(\mathbf{x})$ is short for $a^{4} \square_{\triangle}^{2}(\mathbf{x})$ [recall (32)]. Numerical integration yields

$$
\left(v_{\triangle}\right)^{-2}(0) \approx 18.5 \quad, \quad\left(v_{\triangle}\right)^{-2}\left(a \mathbf{e}_{(i)}\right) \approx-4.43,
$$

so that the lowest correction to the free energy is

$$
-F_{\triangle}^{\text {stress }} / k_{B} T \approx 2 N e^{-9\left(v_{\triangle}\right)^{-2}(0) / 16 \beta_{\triangle}(1+\nu)} .
$$

The next term in the expansion of $-\beta F_{\triangle}^{\text {stress }}$ is about a factor $\exp \left[-9\left(v_{\triangle}\right)^{-2}(0) /(16 \beta \triangle(1+\nu))\right]$ smaller than the lowest-order term in (42). By taking into account the lowest-order result $\beta_{\triangle}(1+\nu) \approx 0.6$ we obtain from this an extremely small factor $e^{-20}$. Thus, the lowest stress configuration of the high-temperature expansion in (42) can be trusted for $\beta_{\triangle}(1+\nu)<10$.

In the case of the square lattice [2] one gets for $Z_{\square}^{\text {stress }}$ the same formula as in (42), except that the range of summation for the gauge field $\chi(\mathbf{x})$ is $\sum_{\chi(\mathbf{x}) \in \mathbb{Z}}$, and that $\left(v_{\triangle}\right)^{-2}$ has to be replaced by $\left(v_{\square}\right)^{-2}$. The numerical values are $\left(v_{\square}\right)^{-2}(0)=20$ and $\left(v_{\square}\right)^{-2}\left(a \mathbf{e}_{x, y}\right)=-8$.

Using the above-derived results we can now calculate the melting temperature from the intersection of the low-temperature expansion $Z_{T \rightarrow 0} Z_{\triangle}^{\text {def }}$ and the hightemperature expansion $Z_{T \rightarrow \infty} Z_{\triangle}^{\text {stress }}$ of the partition 
function $Z$. Instead of (28), we find from the corrections (36) and (42) that the melting point satisfies

$$
\begin{aligned}
\beta(1+\nu) \approx & A \exp \left[-M_{1} e^{-B_{1} \beta(1+\nu)}-M_{2} e^{-B_{2} \beta(1+\nu)}\right] \\
& \times \exp \left[+N e^{-\frac{C}{\beta(1+\nu)}}\right]
\end{aligned}
$$

with $\beta=\beta_{\triangle}$ in the case of the triangular lattice and $\beta=\beta \square$ for the square lattice. The parameters are

\begin{tabular}{c|cc} 
& triangular & square \\
\hline$A$ & 0.51 & 0.81 \\
$M_{1}$ & 4 & 4 \\
$M_{2}$ & 12 & - \\
$N$ & 4 & 4 \\
$B_{1}$ & 6.45 & 6.31 \\
$B_{2}$ & 8.6 & - \\
$C$ & 10.4 & 5 \\
\hline
\end{tabular}

Expression $\tilde{F} / N k_{B} T \equiv F / N k_{B} T+\ln \left((1-\nu)^{1 / 2}(1+\right.$ $\nu)) / N$ is a function of $\beta(1+\nu)$. We show in Fig. 4 the corresponding curves of the low- and high-temperature expansion for the triangular lattice and the square lattice. We obtain for the square lattice that the high and low-temperature curves intersect in one point. In contrast to this, the curves of the triangular lattice do not intersect. This is caused by an enhancement of the defect contributions to the low-temperature expansions for the triangular lattice. The two curves have merely a wouldbe intersection near

$$
\beta_{\triangle}(1+\nu) \approx 0.6
$$

where the distance between both curves on the $\beta(1+\nu)$ axis is approximatively

$$
\Delta \beta_{\triangle}(1+\nu) \approx 0.05 .
$$

We point out that the non-intersecting of the two curves is not reasoned in a failure of our approximations. As estimated above the two curves can be trusted for $0.15<$ $\beta_{\triangle}(1+\nu)<10$.

For a square lattice there was a definite intersection at

$$
\beta_{\square}(1+\nu) \approx 0.8 .
$$

In either case, the higher-order corrections to the melting temperature are small compared to the lowest-order result (28). What is the reasons for the difference in the transition properties of the two lattices? We see from (44) that the numbers $M_{1}$ and $B_{1}$ are almost identical for the triangular and the square lattice. The $\eta$-configuration for the triangular lattice of this term is a triangle with charges $\pm 2 / 3$ shown in Fig. 3. The corresponding $\eta$ configuration for the square lattice is given by a basic square with charges \pm 1 2]. The difference between triangular and the square lattices lies in the term proportional
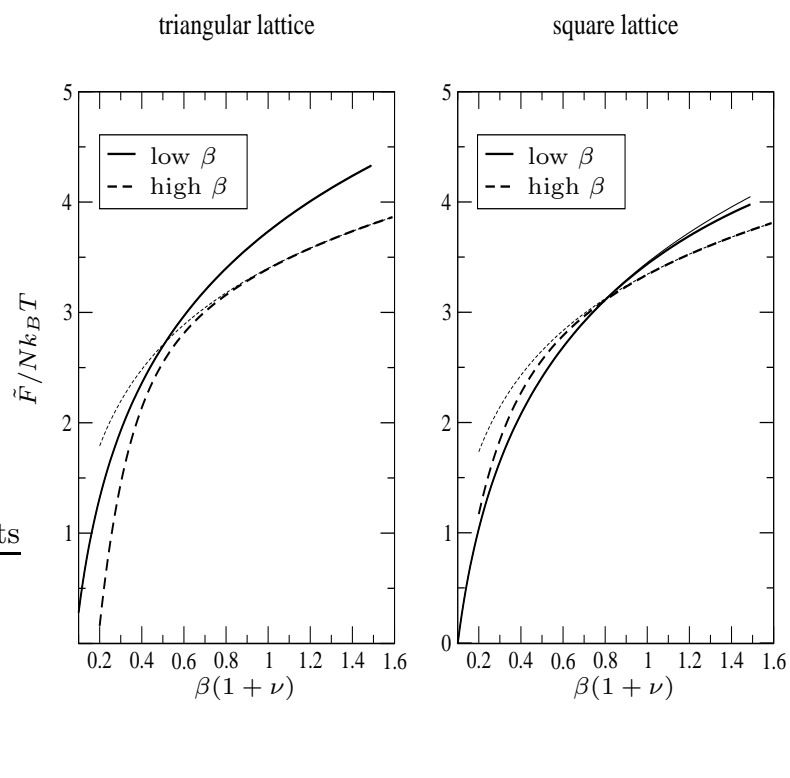

FIG. 4: Low- and high-temperature expansions of $\tilde{F} / N k_{B} T \equiv$ $F / N k_{B} T+\ln \left((1-\nu)^{1 / 2}(1+\nu)\right) / N$ for the triangular lattice and the square lattice. The thin line curves are the lowestorder results of Section V.A, while the thick line curves take into account also the defect and stress contributions of Section V.B. Note that stress corrections are negligible. The low-temperature lowest-order and higher-order curves for the triangular lattice are practically on top of each other.

to $M_{2}$ in (43) whose $\eta$-configuration is shown in Fig. 2(a). A term of this type is absent for square lattices. The defects lie on two neighboring rhombuses of charges $\pm 2 / 3$. The corresponding configuration for the square lattice would consist of two neighboring squares. The distance between the rhombuses is much smaller than that of the two nearby squares. This results in an energy reduction of the corresponding defect configuration $\eta$ for the triangular lattice. This was the reason why this contribution was negligible for square lattices.

From previous work we know that melting of two dimensional crystals can proceed in only two possible scenarios: either by the proliferation of defects as in three dimensions, in which case the transition is of first order, or by a two-step unbinding of defects (KTHNY). It was shown by Saito 31 in Monte Carlo simulations of a gas of dislocations on a triangular lattice and in the textbook [2] by general arguments that the core energies of the dislocations decide which of the two options is chosen. For small core energies, the transition is of first order, for large core energies, the KTHNY scenario prevails. This agrees with the properties of our harmonic defect model. From the long-range behavior of the Coulomb force between the dislocations the unbinding transition will always take place even if the defects are not yet prolific at a temperature given by the universal equation KT criterion. This implies that the $\beta$-value at the melting point must always satisfy the inequality $\beta_{\triangle}(1+\nu) \geq \sqrt{3} / \pi \approx 0.55$ irrespective of the lattice sym- 
metry [14]. If the inequality is almost an equality, which is the case for our result (45), the transition is of the KTHNY type. For square lattices, the situation is different. By comparing the melting temperature of the square (47) and triangular (45) lattice we see that defects proliferate before dislocations can separate. This agrees with the naive expectation that square lattices should be less stable against thermal fluctuations than triangular ones. In the lattice models this is a consequence of the smaller core energies of dislocations on square lattices [2], as we know from Saito's simulation work.

The behavior of the free energy shown in Fig. 4 excludes the single-first-oder scenario for triangular lattices, leaving only the KTHNY scenario. Indeed, for this scenario we cannot expect an intersection of the curves in Fig. 4. The intermediate hexatic phase between the two continuous transitions, which the model should exhibit, would be inaccessible to both low- and high-temperature expansions. With less certainty, we extract an estimate for the separation of the two KT transitions from the distance $\Delta \beta$ of the two nonintersecting curves. Our finding of a KTHNY scenario is in accordance with recent computer simulations for triangular Lennard-Jones as well as electron lattices 21, 22, 23, 24].

The situation for the square lattice is much clearer. There is a definite intersection point in Fig. 4, indicating that the melting transition is of first order, as found by computer simulations [2, 13. . When trying to compare this result with real crystals in nature one has the difficulty that square lattices are hard to produce. They need complicated interparticle forces, and only Weber et al. 30 succeeded to do build such crystals with the help of a three-body potential in a computer model. They found indeed a first-order melting transition in their simulation, in agreement with the prediction in 2, 13], and with the above conclusion.

\section{LENNARD-JONES AND ELECTRON LATTICES IN 2D}

Let us compare our results quantitatively with computer simulations of 2D Lennard-Jones as well as electron lattices. In both cases, the ground state is triangular, due to the simplicity of the interaction potential. In the Lennard-Jones lattice, the interaction potential is

$$
V_{L J}(r)=4 \epsilon\left[\left(\frac{\sigma}{r}\right)^{12}-\left(\frac{\sigma}{r}\right)^{6}\right] .
$$

Frenkel and McTargue 32] carried out an isothermalisochoric molecular dynamics simulation, and observed a hexatic phase in accordance with KTHNY theory. In contrast, Abraham at. al. and others [33, 34] found with the help of both molecular dynamics as well as Monte-Carlo simulations that the melting transition of the Lennard-Jones lattice is of first order. The discrepancy induced simulations on larger systems, which found again KTHNY-like melting transitions [21, 22].

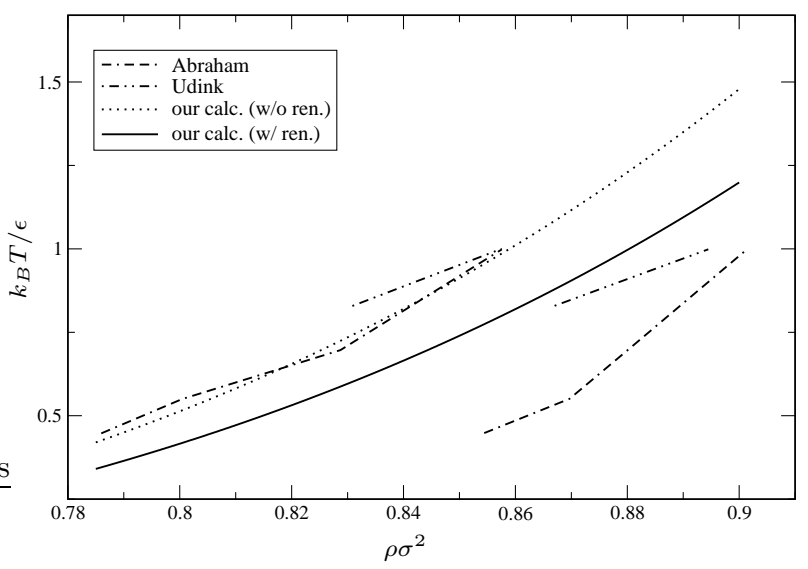

FIG. 5: Reduced melting temperature $k_{B} T_{m} / \epsilon$ of Eq. (51) as a function of the reduced density $\rho \sigma^{2}$. The dotted line denotes the melting temperature calculated with the $T=0$ elastic constants. The solid curve takes the thermal softening of the elastic constants by non-harmonic elasticity into account, calculated in Section VII. For comparison we show the melting temperature obtained by Abraham and Barker et al. 33] and Udink et al. 21] by simulation. The two curves of the simulation enclose the coexisting crystal-liquid region or the hexatic phase, respectively.

To apply our model, we extract from the potential (48) the elastic moduli $\lambda$ and $\mu$ as follows 2 :

$$
\begin{aligned}
& \lambda=\frac{\epsilon}{v}\left[324\left(\frac{\sigma}{a}\right)^{12}-108\left(\frac{\sigma}{a}\right)^{6}\right], \\
& \mu=\frac{\epsilon}{v}\left[180\left(\frac{\sigma}{a}\right)^{12}-36\left(\frac{\sigma}{a}\right)^{6}\right] .
\end{aligned}
$$

Inserting these into our result (45), we obtain for the melting temperature $T_{m}$ of the triangular Lennard-Jones lattice:

$$
\begin{aligned}
\frac{k_{B} T_{m}}{\epsilon} & \approx 112\left(\sigma^{2} \rho\right)^{3}\left[\left(\sigma^{2} \rho\right)^{3}-0.3\right] \frac{1}{0.6(2 \pi)^{2}} \\
& \approx 4.725\left(\sigma^{2} \rho\right)^{3}\left[\left(\sigma^{2} \rho\right)^{3}-0.3\right] .
\end{aligned}
$$

Here $\rho$ is the particle density $1 / v$. Figure 5 shows $k_{B} T_{m} / \epsilon$ as a function of $\sigma^{2} \rho$ in comparison with the two melting curves enclosing the coexisting crystal-liquid region or the hexatic phase, respectively, calculated by Abraham and Barker et al. in 33| getting a first-order transition and by Udink et al. in [21] consistent with KTHNY. The curves are calculated by determining the phase transition line via thermodynamic integration. The results in 21] are in good accordance with the simulations on large systems showing also a KTHNY transition.

Obviously, the value 0.6 in relation (45) is too small to agree with the simulations. This discrepancy will be removed below in Section VII.

Next, we discuss the system of a 2D electron lattice. Wigner 35] predicted in 1934 that a gas of electrons 
should becomes a triangular crystal at low temperature and density. Experimentally, this was first observed by Grimes and Adams 4] for electrons trapped on a liquid helium surface. The electrons interact via the Coulomb potential

$$
V_{e e}(r)=e^{2} / r,
$$

and their kinetic energy is negligible at low temperature and density. As in the case of the Lennard-Jones lattice, the ground state of the system is built of a triangular lattice [12]. Hockney and Brown [36] were the first which carry out a molecular dynamic simulation of this system. They found a $\lambda$-like melting transition. In contrast, Gann et al. and Kalia et al. 37] found, via Monte-Carlo and molecular dynamics studies, a first-order melting transition. Differing from both results, Morf [38] obtained a melting temperature consistent with the KTHNY theory, when he took into account the higher-order nonlinear corrections to the shear modulus. His results were reproduced by more recent simulations on larger electron lattices [23, 24].

The elastic constants for the electron lattice are calculated in Refs. 2, 12]. The Lamé constant is infinite, so that the electron lattice has an infinite modulus of compression. The shear constant $\mu$ is equal to

$$
\mu=\eta e^{2} / v^{3 / 2}, \quad \eta \approx 0.245 .
$$

The simulation data yield for the melting temperature $T_{m}$ the relation

$$
\Gamma_{m} \approx 130 \pm 10,
$$

where $\Gamma$ is the reduced inverse temperature

$$
\Gamma \equiv \sqrt{\pi} e^{2} / \sqrt{v} k_{B} T .
$$

To compare this result with our theory we insert the elastic constants into Eq. (45) and find $\Gamma_{m} \approx 86.4$, which is about $30 \%$ smaller than the simulation value. Also here, we shall remove the discrepancy in Section VII.

Concluding this section we observe that the interval $\Delta \beta_{\triangle}(1+\nu) \approx 0.05$ found in Fig. 4 for the triangular lattice in Eq. (46) agrees with the temperature interval of the intermediate (hexatic) phase seen in the computer simulations of both Lennard-Jones as well as electron lattices [21, 22, 23, 24].

\section{ANHARMONIC CORRECTIONS TO MELTING FORMULA}

The melting temperature found in the last section from formula (45) lies too high. The same problem was encountered by Thouless [39] in his calculation of the melting temperature for the electron lattice, who used the melting formula $\beta_{\triangle}(1+\nu)=\sqrt{3} / \pi \approx 0.55$ where dislocations unbind in the KTHNY theory. His formula is quite close to ours in Eq. (45). It was shown by Morf in [38] that the discrepancy between the prediction with the simulated melting temperature of the electron lattice can be explained by the thermal softening of the elastic constant $\mu$, which he determined with the help of a computer simulation to be [recall [55)]

$$
\mu(T) \approx \mu(0)(1-30.8 / \Gamma),
$$

where $\mu(0)$ is the zero-temperature shear modulus (53). By using (56) one obtains from our formula (45) $\Gamma_{m} \approx$ 117.2, which is now close to Morf's simulation value (54) and in quite excellent agreement with the most recent melting value in Ref. 24 obtained by Monte-Carlo simulation of a large Wigner lattice $\Gamma_{m} \approx 123$.

For 2D Lennard-Jones crystals, the thermal softening can only be estimated theoretically. We obtain a reasonable approximation of the renormalized elastic constants by observing that the thermal softening due to non linear elasticity is much stronger for the transversal than for the longitudinal sound velocity 2]. A further simplification comes from the fact [2] that the renormalization of the transversal sound is proportional to the square root of $\mu$, and has in first approximation a universal low-temperature law depending only on the lattice structure. This yields the approximations

$$
\begin{aligned}
& 2 \mu(T)+\lambda(T) \approx 2 \mu(0)+\lambda(0), \\
& \mu(T) \approx \mu(0)\left[1-c \frac{\mu(0)}{\beta(0)}\left(\frac{1}{\mu(0)}+\frac{1}{2 \mu(0)+\lambda(0)}\right)\right] .
\end{aligned}
$$

The constant $c$ is the same for all lattices with the same structure. Its value can therefore be deduced from (56):

$$
c_{\triangle} \approx 0.11 .
$$

Combining (57) and (58) we obtain

$$
\begin{aligned}
& \mu(T) \approx \mu(0)\left[1-\frac{c}{\beta(0)}\left(\frac{3}{2}-\frac{\nu(0)}{2}\right)\right], \\
& \nu(T) \approx \nu(0)+(1-\nu(0)) \frac{c}{\beta(0)}\left(\frac{3}{2}-\frac{\nu(0)}{2}\right) .
\end{aligned}
$$

Taking this renormalization into account, we obtain from (45) the melting formula for triangular lattices

$$
\begin{aligned}
\beta_{\triangle}(T)(1+\nu(T)) & \approx \beta_{\triangle}(0)(1+\nu(0))-c \nu(0)(3-\nu(0)) \\
& \approx 0.6 .
\end{aligned}
$$

For the Lennard-Jones lattice where $\nu(0) \approx 0.48$, this leads to a melting formula

$$
\beta_{\triangle}(0)(1+\nu(0)) \approx 0.74,
$$

rather than (45), thus lowering the melting temperature by about $20 \%$. The dashed curve in Fig. 5 shows the resulting melting temperature for the Lennard-Jones lattice, which is compatible with the simulation data [21, 33]. It is obtained from (51) by multiplying the righthand side with the softening factor $0.6 / 0.74 \approx 0.81$. 


\section{TWO-DIMENSIONAL LINDEMANN PARAMETER OF MELTING}

The Lindemann parameter 27] expressed in terms of the expectation value of the lattice displacement $\sqrt{\left\langle u^{2}\left(\mathbf{r}_{i}\right)\right\rangle} / a$ is not applicable in two dimensions, where the expectation value diverges due to excessive longwavelength fluctuations of the displacement field. An appropriate modified Lindemann parameter in $2 \mathrm{D}$ is $7,40,41$

$$
\tilde{L}=\sqrt{\left\langle\left[u\left(\mathbf{x}_{i}\right)-u\left(\mathbf{x}_{i+1}\right)\right]^{2}\right\rangle} / a
$$

where $\mathbf{x}_{i}$ and $\mathbf{x}_{i+1}$ are nearest neighbors in the lattice. The correlation function of the displacement field following from the energy (8) is

$$
\begin{aligned}
& \left\langle u_{i}(\mathbf{k}) u_{j}(-\mathbf{k})\right\rangle=N\left(k_{B} T\right) \\
& \times \frac{\bar{K}_{(l)} K_{(m)} \mathbf{e}_{(l)} \mathbf{e}_{(m)} \delta_{i j}-\frac{\lambda+\mu}{\lambda+2 \mu} \bar{K}_{(l)} K_{(m)} e_{(l)} e_{(m)}}{v \mu \frac{4}{9}\left(\bar{K}_{(l)} K_{(m)} \mathbf{e}_{(l)} \mathbf{e}_{(m)}\right)^{2}} .
\end{aligned}
$$

On square lattices, we must merely replace $(2 / 3) K_{(l)}\left(e_{(l)}\right)_{x, y} \rightarrow K_{x, y}$ and $(2 / 3) \bar{K}_{(l)}\left(e_{(l)}\right)_{x, y} \rightarrow \bar{K}_{x, y}$ 2]. From this equation we obtain for both square and triangular lattices the modified Lindemann parameter

$$
\tilde{L}=\frac{1}{4 \pi} \sqrt{\frac{3-\nu(T)}{\beta(T)}} .
$$

Let us compare our Lindemann parameter with that determined by Bedanov and Gadiyak via computer simulations for Lennard-Jones and electron lattices [40]. For the electron lattice we insert the temperature-dependent elastic constants $\mu(T)$ of Eq. (60) and $\nu(T)=\nu(0)=1$ into the melting formula (62), and obtain from (66) $\tilde{L}_{\triangle} \approx 0.20$. The corresponding simulation value of $\mathrm{Be}-$ danov and Gadiyak is $\tilde{L}_{\triangle} \approx 0.17$, quite close to this.

Now we turn to the Lennard-Jones lattice. Using the renormalized elastic constant (61) with $\nu(0) \approx 0.48$ and the melting formula (62) we obtain from (66) the Lindemann parameter $\tilde{L}_{\triangle} \approx 0.20$, again very close to the value $\tilde{L}_{\triangle} \approx 0.18$ found in the simulations of Bedanov and Gadiyak 40].

Hence our theory yields generalized Lindemann parameters for both electron and Lennard-Jones lattices in good agreement with simulation results of Bedanov and Gadiyak [40].

\section{SUMMARY}

We have set up the simplest possible lattice model of defect melting on a two-dimensional triangular lattice. It accounts for the correct elastic fluctuations and by means of discrete-valued defect gauge fields for the fluctuations of dislocations and disclinations. The latter give rise to melting transitions, and the melting temperature follows a formula of the Lindemann type, with a modification due to the two dimensions. The value of the Lindemann parameter is predicted, and agrees with estimates obtained from computer simulations on both Lennard-Jones and electron lattices.

The melting transition is determined from the intersection of the free energies calculated once from a low-temperature expansion and once from a hightemperature expansion. While the square lattice melts in a first-order transition, the curves for the triangular lattice are compatible with two continuous transitions, as predicted by the KTHNY theory and found in computer simulations. The difference is due to the enhancement of the defect fluctuations in comparison to the square lattice caused by the smaller distance between basic nearby rhombuses on triangular lattices, in comparison to the corresponding defect configuration of two nearby squares on the square lattice. The resulting enhancement of smallest defect configurations leads to a decrease of the melting temperature.

\section{APPENDIX A: ELIMINATION OF THE GAUGE DEGREES OF FREEDOM}

In this section we eliminate the gauge degrees of freedom in the $n_{(l m)}$ sum of Eq. (12) enforced by the functional $\Phi\left[n_{(l m)}\right]$. We shall prove the following: The gauge degrees of freedom in (12) with (11) and (13) are fixed in the case that one chooses the gauge fixed integer-valued defect fields (15) with the boundary condition (16) for the bulk defect field $n$.

By using (4) and (13) one can transform (11) to the following expression

$$
\begin{aligned}
\nabla_{x} \mathbf{u}(\mathbf{x}) \rightarrow & -\nabla_{(2)} \mathbf{u}(\mathbf{x})+n_{(2 m)}(\mathbf{x}) \mathbf{e}_{(m)} \\
= & -\nabla_{(2)} \mathbf{u}+\left(\begin{array}{c}
\frac{1}{2} n_{(21)}-n_{(22)} \\
\frac{\sqrt{3}}{2} n_{(21)}
\end{array}\right), \quad(\mathrm{A} 1) \\
\nabla_{y} \mathbf{u}(\mathbf{x}) \rightarrow & \frac{1}{\sqrt{3}}\left(2 \nabla_{(1)}+\nabla_{(2)}\right) \mathbf{u}(\mathbf{x}) \\
& -\frac{1}{\sqrt{3}}\left(2 n_{(1 m)} \mathbf{e}_{(m)}+n_{(2 m)} \mathbf{e}_{(m)}\right) \\
= & \frac{1}{\sqrt{3}}\left(2 \nabla_{(1)}+\nabla_{(2)}\right) \mathbf{u}(\mathbf{x}) \\
& +\frac{1}{\sqrt{3}}\left(\begin{array}{c}
-n_{(11)}+2 n_{(12)}-\frac{1}{2} n_{(21)}+n_{(22)} \\
-\sqrt{3} n_{(11)}-\frac{\sqrt{3}}{2} n_{(21)}
\end{array}\right) .
\end{aligned}
$$

Here we put $n_{(l 3)}=0$ which is possible due to the overcounting of the basis $\mathbf{e}_{(l)}$. Now, we can carry out the substitution of the displacement fields $\mathbf{u}(\mathbf{x}) \rightarrow \mathbf{u}(\mathbf{x})+a \mathbf{N}(\mathbf{x})$ in (A1) where $a \mathbf{N}(\mathbf{x})$ is some displacement field corresponding to a jump from one lattice site to another. We determine it by the requirement that

$$
a \nabla_{(2)} N_{x}(\mathbf{x})=n_{(2 m)} e_{(m)_{x}}=\frac{1}{2} n_{(21)}-n_{(22)}
$$


and

$$
\begin{aligned}
& \frac{a}{\sqrt{3}}\left(2 \nabla_{(1)}+\nabla_{(2)}\right) N_{y}(\mathbf{x}) \\
& =\frac{1}{\sqrt{3}}\left(2 n_{(1 m)} e_{(m)_{y}}+n_{(2 m)} e_{(m)_{y}}\right)=n_{(11)}+\frac{1}{2} n_{(21)} .
\end{aligned}
$$

From these equations we obtain

$$
a \nabla_{(l)} N_{x}(\mathbf{x}) \in \frac{\mathbb{Z}}{2} \quad, \quad a \nabla_{(l)} N_{y}(\mathbf{x}) \in \frac{\sqrt{3}}{2} \mathbb{Z}
$$

and

$$
\left(2 a \nabla_{(2)} N_{x}(\mathbf{x})\right) \bmod 2=\left(\frac{2 a}{\sqrt{3}} \nabla_{(2)} N_{y}(\mathbf{x})\right) \bmod 2 .
$$

To derive the last equation we have used the fact that the half-integer terms on the right hand sides of (A3) and (A4) coincide. We get unique solutions of (A3) and (A4) when fixing $N_{x}(\mathbf{x})$ and $N_{y}(\mathbf{x})$ on one half of the boundary of the system where we now suppose that we have approximately a square sample. Thus, we can for example fix the values of $N_{x}(\mathbf{x})$ and $N_{y}(\mathbf{x})$ on the upper and the rightmost boundary of the sample which we denote by $\mathcal{B}$. Then, due to the periodic boundary conditions for the displacement field $\mathbf{u}$ the values of $N_{x}(\mathbf{x})$ and $N_{y}(\mathbf{x})$ are determined on the whole boundary of the system. Because of the periodic boundary conditions for $N_{x}(\mathbf{x})$ and $N_{y}(\mathbf{x})$ it is not clear that the unique solution of (A3) and (A4) respects these periodic boundary conditions. That this is not generally true can be most easily seen for the square lattice [2]. Whether this is true or not depends on the values of the defect configuration on the right hand sides of (A3), A4 and the boundary values of $N_{x}(\mathbf{x})$ and $N_{y}(\mathbf{x})$ on $\mathcal{B}$.

The values of the field $n(\mathbf{x})$ in (15) or (17) are now given by the following expression

$$
\begin{aligned}
n(\mathbf{x})= & \frac{\sqrt{3}}{2}\left[a\left(\nabla_{x} N_{y}(\mathbf{x})+\nabla_{y} N_{x}(\mathbf{x})\right)\right. \\
& \left.+n_{(2 m)} e_{(m)_{y}}-\frac{1}{\sqrt{3}}\left(2 n_{(1 m)}+n_{(2 m)}\right) e_{(m)}\right] \\
= & -2 n_{(11)}+n_{(12)} \\
& +a \nabla_{(1)} N_{x}(\mathbf{x})+a \sqrt{3} \nabla_{(1)} N_{y}(\mathbf{x})
\end{aligned}
$$

Here we take into account that the Hamiltonian (7) depends on the lattice derivates of the displacement field only in the strain combination $\nabla_{x} u_{y}+\nabla_{y} u_{x}$. We now observe that $\nabla_{(1)} N_{x, y}(\mathbf{x})$ can be written as a linear combination of $\nabla_{(2)} N_{x, y}\left(\mathbf{x}^{\prime}\right)$ with $y^{\prime}=y$ or $y^{\prime}=y+e_{(1)}$ and a lattice derivate corresponding to a rightmost boundary edge. From this, (A5), we obtain that $n(\mathbf{x})$ on the whole lattice, given by the right-hand side of (A7), is integer valued when $n(\mathbf{x})$ on the boundary $\mathbf{x} \in \mathcal{B}$ have integer values.

The boundary values $N_{x}(\mathbf{x})$ and $N_{y}(\mathbf{x})$ on $\mathcal{B}$ are fixed by choosing the boundary conditions (16) for $n(\mathbf{x})$. The periodic boundary conditions for $N_{x}(\mathbf{x})$ and $N_{y}(\mathbf{x})$ on $\mathcal{B}$ can be fixed when taking into account A1 and A2 only for $\mathbf{x} \neq \mathcal{B}$. From a1 and A2 we obtain further that we can fix $n_{(22)}(\mathbf{x})=0$ on $\mathcal{B}$.

As a result we obtain the gauge-fixed path integral (14) with (15) and (16) for the triangular lattice which takes into account all defect degrees of freedom.
[1] W. Shockley, In L 'Etat Solide, Proceedings of Neuvienne-Consail de Physique, Brussels, 1952, Ed. R. Stoops, Solvay Institute de Physique, Brussels, Belgium

[2] H. Kleinert, Gauge Fields in Condensed Matter, Vol. II Stresses and Defects: Differential Geometry, Crystal Melting, World Scientific, Singapore, 1989 (readable online at www.physik.fu-berlin.de/ ${ }^{\sim k l e i n e r t / r e . h t m l \# b 2) . ~}$

[3] H. Kleinert, In Progress in Gauge Field Theory, ed. by G. 't. Hooft et al., Plenum Press 1984, pp 373-401. (www.physik.fu-berlin.de/ ${ }^{\mathrm{k} l e i n e r t / r e 2 . h t m l \# 118) . ~}$

[4] C. G. Grimes and G. A. Adams, Phys. Rev Lett. 42, 795 (1979).

[5] P. A. Heiney, R. J. Birgeneau, G. S. Brown, P. M. Horn, D. E. Moncton, and P. W. Stephens, Phys. Rev. Lett. 48, 104 (1982).

[6] C. A. Murray and D. H. Van Winkle, Phys. Rev. Lett. 58, 1200 (1987).

[7] K. Zahn, R. Lenke, and G. Maret, Phys. Rev. Lett. 82, 2721 (1999); J. Zanghellini, P. Keim, and H. H. von Grüenberg, J. Phys.: Condens. Matter 17, 3579 (2005).

[8] P. L. Gammel, A. F. Hebard and D. J. Bishop, Phys.
Rev. Lett. 60, 144 (1988).

[9] B. J. Alder and T. E. Wainwright, Phys. Rev. 127, 359 (1962); H. Watanabe, S. Yukawa, Y. Ozeki, and N. Ito, Phys. Rev. E 66, 041110 (2002).

[10] F. H. Stillinger and T. A. Weber, J. Chem. Phys. 74, 4015 (1981); T. A. Weber and F. H. Stillinger, ibid 74, 4020 (1981).

[11] F. F. Abraham, Phys. Rev. B 23, 6145 (1981).

[12] L. Bonsall and A. A. Maradudin, Phys. Rev. B 15, 1959 (1977).

[13] W. Janke and H. Kleinert, Phys. Lett. A 114, 255 (1986) (www.physik.fu-berlin.de/ ${ }^{\sim}$ kleinert/re2.html\#135); W. Janke and D. Toussain, Phys. Lett. A 116, 387 (1986).

[14] B. I. Halperin and D. R. Nelson, Phys. Rev. Lett. 41, 121 (1978); D. R. Nelson and B. I. Halperin, Phys. Rev. B 19, 2457 (1979); A. P. Young, Phys. Rev. B 19, 1855 (1979).

[15] S. T. Chui, Phys. Rev. Lett. 48, 933 (1982).

[16] T. V. Ramakrishnan, Phys. Rev. Lett. 48, 541 (1982).

[17] H. Kleinert, Phys. Lett. A 89, 294 (1982). (www.physik.fu-berlin.de/ ${ }^{\sim k l e i n e r t / r e 1 . h t m l \# 89) ; ~}$ 
[18] H. Kleinert, Phys. Lett. A 130, 443 (1988) (www .physik.fu-berlin.de/ ${ }^{\mathrm{k} l e i n e r t / r e 2 . h t m l \# 174) ; ~}$ W. Janke and H. Kleinert, Phys. Rev. Lett. 61, 2344 (1988) (www.physik.fu-berlin.de/ ${ }^{\sim k l e i n e r t / ~}$ re2.html\#179); H. Kleinert, Phys. Lett. A 136, 468 (1989) (www.physik.fu-berlin.de/ $\left.{ }^{\sim} k l e i n e r t / 183\right)$.

[19] J. M. Kosterlitz and D. J. Thouless, J. Phys. C 6, 1181 (1973).

[20] A. Thomy and X. Duval, J. Chem. Phys. 67, (1970) 1101.

[21] C. Udink and J. van der Elsken, Phys. Rev. B 35, 279 (1987).

[22] K. Chen, T. Kaplan, and M. Mostoller, Phys. Rev. Lett 74, 4019 (1995); F. L. Somer, Jr., G. S. Canright, T. Kaplan, K. Chen, and M. Mostoller, Phys. Rev Lett. 79, 3431 (1997); F. L. Somer, Jr., G. S. Canright, and T. Kaplan, Phys. Rev E 58, 5748 (1998); A. C. Mitus, A. Z. Patashinski, A. Patrykiejew, and S. Sokolowski Phys. Rev. B 66, 184202 (2002).

[23] S. Muto and H. Aoki, Phys. Rev. B 59, 14911 (1999).

[24] W. J. He, T. Cui, Y. M. Ma, Z. M. Liu, and G. T. Zou, Phys. Rev. B 68, 195104 (2003).

[25] H. Kleinert, Phys. Lett. A 95, 381; 493 (1983) (www.physik.fu-berlin.de/ ${ }^{\sim} k l$ einert/re2.html\#101; 104).

[26] H. Kleinert and Y. Jiang, Phys. Lett. A 313, 152 (2003) (www.physik.fu-berlin.de/ ${ }^{k}$ leinert/re3.html\#340).

[27] F. A. Lindemann, Phys. Z. 11, 609 (1910).

[28] L. D. Landau and E. M. Lifshitz, Electrodynamics of Continous Media, Oxford, Pergamon Press, 1960 (Course of theoretical physics 8).

[29] T. Inui, Y. Tanabe, Y. Onodera, Group Theory and Its
Applications in Physics, Springer Verlag, Berlin Heidelberg, 1996.

[30] T. A. Weber and F. H. Stillinger, Phys. Rev. E 48, 4351 (1993).

[31] Y. Saito, Phys. Rev. Lett. 48, 1114 (1982); Phys. Rev. B 26, 6239 (1982).

[32] D. Frenkel and J. P. McTague, Phys. Rev. Lett. 42, 1632 (1979).

[33] F. F. Abraham, Phys. Rep. 80, 339 (1981); J. A. Barker, D. Henderson, and F .F. Abraham, Physica 106A, 226 (1981).

[34] S. W. Koch and F. F. Abraham, Phys. Rev. B 27, 2964 (1983); J. Tobochnik and G. V. Chester, Phys. Rev. B 25, 6778 (1982); K. J. Strandburg, J. A. Zollweg, and G. V. Chester, Phys. Rev. B 30, 2755 (1984); S. Toxvaerd, Phys. Rev. A 24, 2735 (1981); A. F. Bakker, C. Bruin, and H. J. Hilhorst, Phys. Rev. Lett. 52, 449 (1984).

[35] E. Wigner, Phys. Rev. 46, 1002 (1934).

[36] R. W. Hockney and T. R. Brown, J. Phys. C 8, 1813 (1975).

[37] R. C. Gann, S. Chakravarty, and G. V. Chester, Phys. Rev. B 20, 326 (1979); R. K. Kalia, P. Vashishta, and S. W. de Leeuw, Phys. Rev. B 23, 4794 (1981).

[38] R. H. Morf, Phys. Rev. Lett. 43, 931 (1979).

[39] D. J. Thouless, J. Phys. C 11, L189 (1978).

[40] V. M. Bedanov and G. V. Gadiyak, Phys. Lett. 109A, 289 (1985).

[41] K. Zahn and G. Maret, Phys. Rev. Lett. 85, 3656 (2000). 